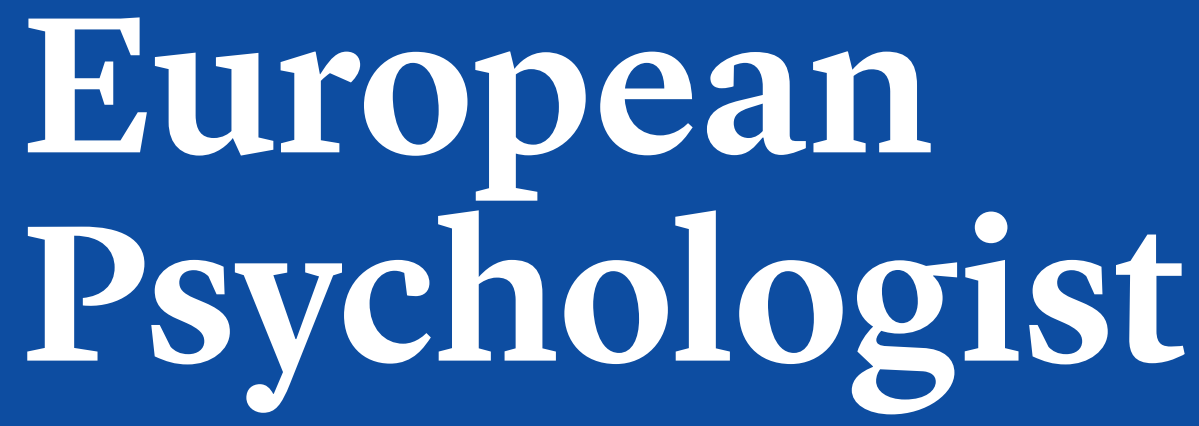

\section{Editor-in-Chief}

Peter Frensch

\section{Managing Editor}

Kristen Lavallee

\section{Associate Editors}

Rainer Banse

Ulrike Ehlert

Katariina Salmela-Aro
Official Organ of the European Federation of Psychologists'Associations (EFPA)

\section{Special Issue}

Noninvasive Brain Stimulation 


\section{Contents}

\section{Special Issue: Noninvasive Brain Stimulation}

(Coordinator: Carlo Miniussi, Guest Editor: Giovanni Galfano)

Editorial

Original Articles and Reviews
A Foreword on the Use of Noninvasive Brain Stimulation in Psychology

1

Carlo Miniussi

Application of Transcranial Electric Stimulation (tDCS, tACS, tRNS):

4

From Motor-Evoked Potentials Towards Modulation of Behaviour

Walter Paulus, Michael A. Nitsche, and Andrea Antal

tES Stimulation as a Tool to Investigate Cognitive Processes in Healthy Individuals

Michal Lavidor

Social Psychology and Noninvasive Electrical Stimulation: A Promising Marriage

Paulo S. Boggio, Gabriel G. Rêgo, Lucas M. Marques, and Thiago L. Costa

Noninvasive Brain Stimulation for the Study of Memory Enhancement in Aging

David Bartrés-Faz and Didac Vidal-Piñeiro

Transcranial Electrical Stimulation in Post-Stroke Cognitive

Rehabilitation: Where We Are and Where We Are Going

Silvia Convento, Cristina Russo, Luca Zigiotto, and Nadia Bolognini

Transcranial Direct Current Stimulation as a Novel Method

65

for Enhancing Aphasia Treatment Effects

Jennifer T. Crinion

Transcranial Electrical Stimulation (tES) for the Treatment

78

of Neuropsychiatric Disorders Across Lifespan

Carolina Pérez, Jorge Leite, Sandra Carvalho, and Felipe Fregni

News and Announcements: From the EFPA Network of National News

Correspondents

Meeting Calendar

98 


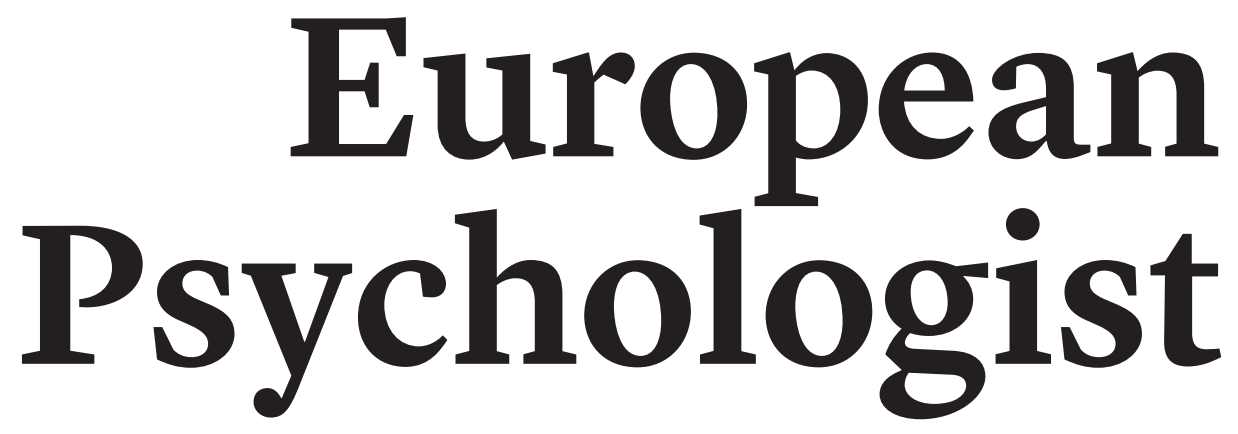

Your article has appeared in a journal published by Hogrefe Publishing. This e-offprint is provided exclusively for the personal use of the authors. It may not be posted on a personal or institutional website or to an institutional or disciplinary repository.

If you wish to post the article to your personal or institutional website or to archive it in an institutional or disciplinary repository, please use either a pre-print or a post-print of your manuscript in accordance with the publication release for your article and our "Online Rights for Journal Articles" (www.hogrefe.com/journals). 


\title{
A Foreword on the Use of Noninvasive Brain Stimulation in Psychology
}

\author{
Carlo Miniussi ${ }^{1,2}$

\begin{abstract}
${ }^{1}$ Neuroscience Section, Department of Clinical and Experimental Sciences, University of Brescia, Italy, ${ }^{2}$ Cognitive Neuroscience Section, IRCCS Centro San Giovanni di Dio Fatebenefratelli, Brescia, Italy
\end{abstract}

"After a small shock to the head, you can achieve happiness, a high mark in school, or the record in your preferred video game." I am reading this claim in the newspaper while sitting on the train going to work. I know what they are talking about; this is my field of research! Even so, I find these words persuasive, and I would like to try these shocks. The train has slowed down, a voice announces my stop, and I am back to a daily reality where small shocks have become a significant tool in basic and clinical neuroscience but certainly not to improve the performance of normal people in their everyday activities.

In recent years, there has been an exponential rise in the number of studies employing noninvasive brain stimulation (NIBS) as a means of gaining understanding of the neural substrates that underlie behavior and as a co-adjuvant for the clinician in treating brain dysfunctions following psychological, cognitive, and neurological disease. In this context, some studies have even suggested that NIBS may enhance brain function in healthy individuals. These findings have attracted media attention and changed the demands of patients and the public to their clinical advisers. Therefore, it is important to open a discussion regarding the utility, effectiveness, and usability of NIBS in real life, outside the laboratory. Along the same lines, the psychologist should understand these techniques to correctly address their patients' demands.

Upon closer examination, we uncover a scenario different from that depicted by the media when NIBS is used to enhance cognition in normal individuals. In this case, the results are confined only to small improvements obtained in the laboratory, improvements that are essential to establish the role of a brain area in a given experimental task, but that certainly do not improve functionality in healthy individuals. Therefore, it is important to prevent pseudoscience from getting ahead of the actual science, which can be done only by discussing the real data. The aim of this special issue is to provide a realistic picture of the state-of-the-art procedures in the field and highlight how these emerging techniques for noninvasive manipulation of the human brain contribute to the development of the field of psychology. Given these premises, it has also become very important to spread this knowledge outside the experimental laboratories and see, if the use of stimulation techniques as a new experimental and therapeutic tool can be fully included in the armamentarium of the psychologist.

This special issue consists of seven papers, all on the use of transcranial stimulation in both healthy and clinical populations. In this framework, the recent advances made due to NIBS methods (Paulus, Nitsche, \& Antal, 2016) in the understanding of the "psychological brain" (Boggio, Rêgo, Marques, \& Costa, 2016; Lavidor, 2016), and whether the use of these brain stimulation techniques alone or combined with rehabilitation, or psychotherapeutic procedures can lead to performance enhancements in the state of our patients are discussed (Bartrés-Faz \& Vidal-Piñeiro, 2016; Convento, Russo, Zigiotto, \& Bolognini, 2016; Crinion, 2016; Pérez, Leite, Carvalho, \& Fregni, 2016).

NIBS includes several methods that can be mainly divided into repetitive transcranial magnetic stimulation (rTMS) and transcranial electrical stimulation (tES). These different NIBS techniques affect neuronal states through different means. However, the stimulation of the human brain requires that these methods are able to induce a change in the membrane potential of neurons. Moreover, the manner in which they achieve this result and the intensity of their action differ among the different NIBS techniques.

Transcranial magnetic stimulation is primarily a method of neurostimulation that includes the induction of depolarization of neuronal membranes and the initiation of action potentials in the area stimulated by electromagnetic induction. This effect is produced using bulky and expensive machinery.

Transcranial electrical stimulation is essentially a method of neuromodulation that uses a smaller and less expensive device. Low-intensity electrical stimulation 
induces a state change in the membrane potential, thereby altering the ionic fluxes. This alteration can result in hyperpolarization or depolarization of the neuron. Transcranial electrical stimulation does not provide direct induction of action potentials, but rather a variation in the response threshold of stimulated neurons, resulting in a modulation of the response that the neurons can provide. Moreover, tES has been proven to be a safe approach, provided that it is administered by trained personnel and with the appropriate medical device (Fregni et al., 2015; Fertonani, Ferrari, \& Miniussi, 2015). While a considerable amount of data is now available to support the safety of electric and magnetic stimulation, we should be aware that some real risks in their usage exist. In particular, TMS should be performed according to current safety guidelines (Rossi, Hallett, Rossini, Pascual-Leone, \& Safety of TMS Consensus Group, 2009).

Given the prevalence of tES methods among psychologists, this issue will mainly address the developments of tES rather than TMS. The introductory article by Paulus et al. (2016) reports the basic aspects of different tES modalities such as transcranial direct current (tDCS), alternating current (tACS), and random noise (tRNS) stimulation. Descriptions of the mechanisms of action for the different approaches are reported, illustrating how several parameters can change the final induced effects. The background knowledge for understanding the basic aspects of tES methods and how to apply them are described, highlighting the relevant features that should be considered when using tES. This work is followed by a description of the possible application of tES as a tool to investigate cognitive processes in healthy individuals by Lavidor (2016). It has been reported that due to tES properties it is possible to interact with the functioning of a specific brain area and therefore the process performed by that area. Such tES-induced modifications can lead to facilitation or impairment of individual performance. In general, it is not important if the induced effect is facilitation or interference, as the unique feature of tES is its ability to interact transiently with the area of the brain stimulated, thus modifying the activity of that area and allowing evaluation of its function. Instead, the induction of behavioral facilitation is important in the field of neurorehabilitation (see Bartrés-Faz \& VidalPiñeiro, 2016; Convento et al., 2016; Crinion, 2016). This approach is expanded and further implemented by Boggio and collaborators (2016), who show how the fields of social neuroscience and psychology have made substantial advances due to the introduction of techniques such as tES, allowing not only the establishment of causal brainbehavior relationships, but also relevance in refining and integrating the theoretical models available to account for different social processes. In particular, it is reported how tES has been used to investigate social pain, social interaction, prejudice, and social decision making. The authors conclude that such applications are highly promising, and they show that tES is indeed an appropriate tool for the further development of this field.

The article by Bartrés-Faz and Vidal-Piñeiro (2016) builds an important bridge, showing how it is possible to use NIBS as an adjuvant therapeutic strategy in the management of age-associated memory decline. Indeed, an area of research that has a great potential for the future is the possibility to maintain or improve memory in aging human beings, given the impact of age-related cognitive dysfunction on the quality of life. Bartrés-Faz and VidalPiñeiro (2016) summarize currently available evidence of memory enhancement and suggest that the use of NIBS offers an attractive and promising opportunity. However, the most important issue is that its use requires appropriate knowledge coupled with a clear understanding of the neurophysiology and cognitive neuroscience of aging. Only by ensuring that these requirements are in place we can refine the hypothesis and select the best procedures to optimize the effect of NIBS on cognition. Such an approach is a key aspect that should also be underscored when NIBS methods are used in the clinical context, as in post-stroke rehabilitation. Most research efforts thus far have been directed in this field, and the results seem, again, very promising. A clear and up-to-date view of using tES in poststroke patients for cognitive rehabilitation is included in the articles by Convento and collaborators (2016) and Crinion (2016). Both of these reports show the important therapeutic potential offered by this technique and provide some crucial suggestions for the design of future clinical trials.

Convento et al. (2016) present the current state-ofthe-art information concerning the use of tES for the improvement of post-stroke visual and cognitive deficits. The evidence supporting the potential of tES to increase neuroplasticity in the adult human brain is reported, and how tES can be used as an adjuvant tool for cognitive rehabilitation is explained. This paper provides a rationalized framework for the important elements that should be considered when using tES. Moreover, it is emphasized that these approaches will not only offer a potentiation of the existing treatments, but will provide novel clues to the factors that may predict a patient's response via novel characterization of the injured cognitive system.

The work by Crinion (2016) discusses the latest studies using tES for enhancing aphasia treatment effects in subacute and chronic post-stroke patients and primary progressive aphasia patients. Importantly, the author shows that before treatment is initiated, patients who are likely to respond to specific tES methods and speech and language therapy should be identified by characterizing the brain state via neuroimaging and specific cognitive evaluations. As for all treatments, in tES, it should not be assumed that one size fits all, and therefore, the final significant effect of training will depend on which cortical areas are targeted by the stimulation and by the training. Therefore, an additional key element is related to the behavioral treatment; applying tES during a non-efficient behavioral intervention will not magnify results that are not present. Therefore, choosing the adequate training task is the most relevant element for treatment success (Crinion, 2016).

Finally, the paper by Pérez et al. (2016) shows that tES can be used to reduce symptoms in a variety of neuropsychiatric conditions, such as depression, schizophrenia, anxiety, autism, and craving, making tES an important complement for psychological/psychiatric disorders. The authors highlight once more the importance of many variables that determine the final effect. There are several 
variables, and each one requires full awareness of its importance. For example, one variable is age; the human brain undergoes several anatomical and functional changes across the life span, and these changes should be considered when we define the technical variables of our protocol. Therefore, tES-induced effects are not only different in different pathologies, but may even be different across the life span (Pérez et al., 2016).

The main aspect that it is highlighted in this special issue is that NIBS can change cortical excitability; nevertheless, such a change is not directly mapped as a unidirectional change in behavior. Applying an electric field to a nonlinear dynamic system, such as the brain, seems likely to have many nontrivial effects that preclude a simple extrapolation on behavior (Miniussi, Harris, \& Ruzzoli, 2013). Therefore, before using these methods, we must have a clear theoretical framework and some methodological knowledge that will allow us to make a clear prediction of the final result.

Overall, one key aspect that emerges is that the final result induced by neurostimulation can be defined only by fully considering the state of the neural system. Basically, we should not forget a classic rule of physiology, which states that we cannot consider the effects induced by tES as pure because the effects induced in the area that we plan to stimulate will depend on the state of activation of the area at the time of stimulation. Consequently, researchers should be aware that the effects of tES are proportional to the level of neuronal activation (i.e., activity dependency) during the application of the stimulation. As described above, tES is a neuromodulatory approach, and therefore, it produces a rather subtle modulatory effect on neural activity. Thus, tES is inadequate to directly induce action potentials in neurons unless neurons are already close to the discharge threshold. In such a context, we can claim that tES is more effective in brain networks that are already selectively engaged by a given $\operatorname{cog}$ nitive task than in networks in a resting state.

Theoretical and technical difficulties have been encountered using NIBS to obtain reliable results and appropriate models to frame them, and there is still a long way to go in the field before well-defined shared approaches will be used. However, by virtue of recent discoveries and developments, important foundations have been built, and future work will build upon this solid foundation. I therefore believe that the excellent contributions of this special issue will provide inspiration for important advances in this field.

\section{References}

Bartrés-Faz, D., \& Vidal-Piñeiro, D. (2016). Noninvasive brain stimulation for the study of memory enhancement in aging. European Psychologist, 21, 41-54. doi: 10.1027/1016-9040/ a000241

Boggio, P. S., Rêgo, G. G., Marques, L. M., \& Costa, T. L. (2016). Social Psychology and noninvasive electrical stimulation: A promising marriage. European Psychologist, 21, 30-40. doi: 10.1027/1016-9040/a000247

Convento, S., Russo, C., Zigiotto, L., \& Bolognini, N. (2016). Transcranial electrical stimulation in post-stroke cognitive rehabilitation: Where we are and where we are going.
European Psychologist, 21, 55-64. doi: 10.1027/1016-9040/ a000238

Crinion J. T. (2016). Transcranial direct current stimulation as a novel method for enhancing aphasia treatment effects. European Psychologist, 21, 65-77. doi: 10.1027/10169040/a000254

Fertonani, A., Ferrari, C., \& Miniussi, C. (2015). What do you feel if I apply transcranial electric stimulation? Safety, sensations and secondary induced effects. Clinical Neurophysiology, 126, 2181-2188. doi: 10.1016/j.clinph.2015.03.015

Fregni, F., Nitsche, M. A., Loo, C. K., Brunoni, A. R., Marangolo, P., Leite, J., . . Bikson, M. (2015). Regulatory considerations for the clinical and research use of transcranial direct current stimulation (tDCS): Review and recommendations from an expert panel. Clinical Research and Regulatory Affairs, 32, 22-35. doi: 10.3109/10601333.2015.980944

Lavidor, M. (2016). tES stimulation as a tool to investigate cognitive processes in healthy individuals. European Psychologist, 21, 15-29. doi: 10.1027/1016-9040/a000248

Miniussi, C., Harris, J. A., \& Ruzzoli, M. (2013). Modelling non-invasive brain stimulation in cognitive neuroscience. Neuroscience \& Biobehavioural Reviews, 37, 1702-1712.

Paulus, W., Nitsche, M. A., \& Antal, A. (2016). Application of transcranial electric stimulation (tDCS, tACS, tRNS): From motor-evoked potentials towards modulation of behaviour. European Psychologist, 21, 4-14. doi: 10.1027/1016-9040/ a000242

Pérez, C., Leite, J., Carvalho, S., \& Fregni, F. (2016). Transcranial electrical stimulation (tES) for the treatment of neuropsychiatric disorders across lifespan. European Psychologist, 21, 78-95. doi: 10.1027/1016-9040/a000252

Rossi, S., Hallett, M., Rossini, P. M., \& Pascual-Leone, A., Safety of TMS Consensus Group. (2009). Safety, ethical considerations, and application guidelines for the use of transcranial magnetic stimulation in clinical practice and research. Clinical Neurophysiology, 120, 2008-2039.

About the author

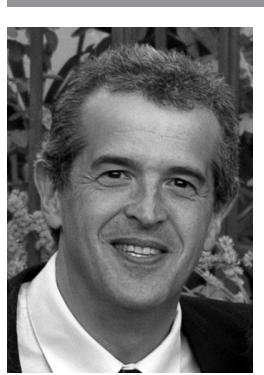

Carlo Miniussi is Full Professor of Human Neurophysiology, Department of Clinical and Experimental Sciences, School of Medicine, University of Brescia (Italy) and Head of the Cognitive Neuroscience Section, Saint John of God Clinical Research Centre - IRCCS Centro San Giovanni di Dio Fatebenefratelli, Brescia (Italy). The core of his research activity is driven by the goal to understand if cortical plasticity can be induced and manipulated by means of noninvasive brain stimulation in healthy and pathological adult brains, to understand the relation between induced synaptic plasticity and cognitive plasticity, and how cognitive plasticity can be sustained by the activity of a functional neuronal network.

Carlo Miniussi

Dept of Clinical and Experimental Sciences

University of Brescia

Viale Europa, 11

25123 Brescia

Italy

Tel. +39030 3717-441

Fax +39030 3717-443

E-mail carlo.miniussi@cognitiveneuroscience.it 